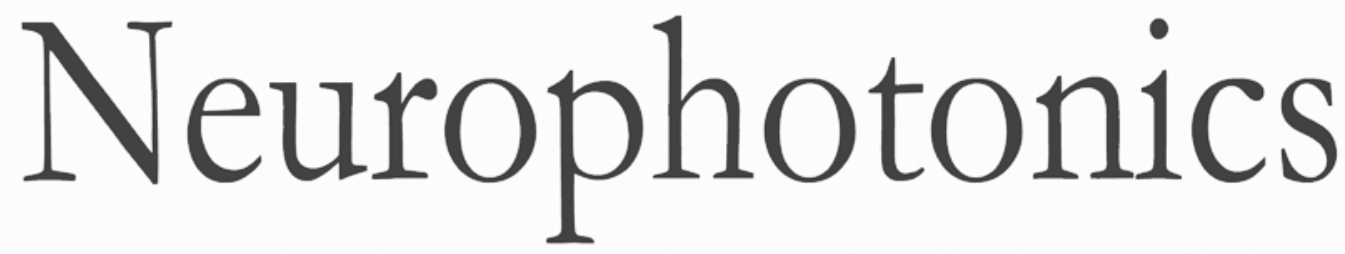

\title{
Quantitative in vivo dual-color bioluminescence imaging in the mouse brain
}

Markus Aswendt

Stefanie Vogel

Cordula Schäfer

Amit Jathoul

Martin Pule

Mathias Hoehn 


\title{
Quantitative in vivo dual-color bioluminescence imaging in the mouse brain
}

\author{
Markus Aswendt, ${ }^{\mathrm{a}, \mathrm{b}, *}$ Stefanie Vogel, ${ }^{\mathrm{a}}$ Cordula Schäfer, ${ }^{\mathrm{a}}$ Amit Jathoul, ${ }^{\mathrm{c}}$ Martin Pule, ${ }^{\mathrm{d}}$ and Mathias Hoehn ${ }^{\mathrm{a}, \mathrm{e}}$ \\ ${ }^{a}$ Max Planck Institute for Metabolism Research, In-vivo-NMR Laboratory, Cologne, Germany \\ bUniversity of Cologne, Faculty of Medicine and University Hospital Cologne, Department of Neurology, Cologne, Germany \\ ${ }^{\circ}$ Cardiff School of Biosciences, Molecular Biosciences, Cardiff, United Kingdom \\ dUniversity College London, Cancer Institute, Department of Haematology, London, United Kingdom \\ 'Leiden University Medical Center, Department of Radiology, Leiden, The Netherlands
}

\begin{abstract}
Bioluminescence imaging $(B L I)$ is an optical imaging method that can be translated from the cell culture dish in vitro to cell tracking in small animal models in vivo. In contrast to the more widely used fluorescence imaging, which requires light excitation, in BLI the light is exclusively generated by the enzyme luciferase. The luciferase gene can be engineered to target and monitor almost every cell and biological process quantitatively in vitro and even from deep tissue in vivo. While initially used for tumor imaging, bioluminescence was recently optimized for mouse brain imaging of neural cells and monitoring of viability or differentiation of grafted stem cells. Here, we describe the use of bright color-shifted firefly luciferases (Flucs) based on the thermostable x5 Fluc that emit red and green for effective and quantitative unmixing of two human cell populations in vitro and after transplantation into the mouse brain in vivo. Spectral unmixing predicts the ratio of luciferases in vitro and a mixture of cells precisely for cortical grafts, however, with less accuracy for striatal grafts. This dual-color approach enables the simultaneous visualization and quantification of two cell populations on the whole brain scale, with particular relevance for translational studies of neurological disorders providing information on stem cell survival and differentiation in one imaging session in vivo. ( ) The Authors. Published by SPIE under a Creative Commons Attribution 4.0 Unported License. Distribution or reproduction of this work in whole or in part requires full attribution of the original publication, including its DOI. [DOI: 10.1117/1.NPh.6.2.025006]
\end{abstract}

Keywords: spectral unmixing; luciferase; bioluminescence; stem cells; implantation; optical imaging.

Paper 18054RR received Sep. 20, 2018; accepted for publication Apr. 15, 2019; published online May 7, 2019.

\section{Introduction}

Firefly luciferases (Flucs) are very efficient molecular and biochemical tools to track cells and proteins and to monitor gene expression in living organisms. ${ }^{1}$ While the first bioluminescence imaging (BLI) was limited to tumor applications, ${ }^{2}$ the firefly gene and the detection hardware were continuously optimized to monitor other cell types, such as stem cells and neurons, in deep tissue with higher or equal sensitivity and specificity compared to, for example, fluorescence imaging. ${ }^{3-8}$ BLI has some unique advantages such as the light is produced directly in the Fluc-expressing cells without the need of an excitation source, promoting a very low background and high signal-tonoise ratios. The efficiency of in vivo light production enables the detection of a minimal number of 1500 to 3000 neural stem cells (NSCs) engrafted to the mouse brain, through the intact skull, connecting tissue, and skin. ${ }^{9,10}$ The survival rate of the transplanted cells, which is an important factor to determine the outcome of translational stem cell studies, can be derived from the quantitative BLI signal, which requires adenosine triphosphate for the luciferase enzyme reaction. ${ }^{11}$ Furthermore, the luciferase gene was engineered to monitor the stem cell fate, for example, the differentiation of stem cells into early neurons in vivo. ${ }^{12}$ However, these measurements can be performed to-date only in separate, single-color experiments. Multicolor BLI, the simultaneous imaging of two or more luciferases with distinct emission spectra, emerged first for in vitro assays and later for imaging bacteria or tumor cells in vivo. ${ }^{13-16}$ Spectral

*Address all correspondence to Markus Aswendt, E-mail: markus.aswendt@ uk-koeln.de unmixing, an algorithm that can distinguish the spectral signatures, is applied to extract the signal from two luciferases emitting light at different wavelengths and to calculate the respective contribution of each reporter on every pixel of an image. ${ }^{16,17}$

The aim of this study was to probe spectral unmixing for bioluminescence neuroimaging with a focus on cell transplantation and to quantify the reliability of dual-color measurements. We have described experiments with a bright red-shifted ${ }^{18,19}$ and a novel green-shifted point mutant of $\mathrm{x} 5$, a thermostable variant of wild-type (WT) Fluc but with higher quantum yields. ${ }^{20,21}$ This dual-color pair was chosen based on a comparison to other commonly used red/green Fluc mutants which showed efficient spectral unmixing in a cell assay in vitro. Furthermore, we have provided, for the first time, a quantitative in vivo discrimination and unmixing-based estimation of two human NSC populations in the mouse brain. Such multiplexed bioluminescence approaches will be useful in monitoring the interaction of multiple cell populations in vivo and in facilitating quantitative dual-color neuroimaging.

\section{Results}

\subsection{In Vitro Spectral Unmixing}

Previously described variations of WT Fluc containing five mutations (F14R, L35Q, V182K, I232K, and F465R) ${ }^{20}$ that reduced the surface hydrophobicity and conferred significant thermostability were further mutated to red-shift (S284T) mutant $\times 5$ red $(\times 5 r)^{18}$ and to produce a novel blue-shifted (V241I/G246A/F250S) mutant x5green (x5g) bioluminescence spectra. In vitro, the dual-color Fluc pair $\mathrm{x} 5 \mathrm{~g} / \mathrm{r}$ was tested against the bioluminescence spectra of WT Fluc (Promega, 
Madison, Wisconsin), PpyRE9 Fluc mutant, ${ }^{22}$ click beetle luciferase CBG99, ${ }^{23}$ and Renilla luciferase hRluc ${ }^{24}$ were acquired with the IVIS Spectrum CT system equipped with 18 emission filter (20-nm bandpass) at $37^{\circ} \mathrm{C}$. Each luciferase was expressed by transfection of lentiviral vectors in the human tumor cell line HEK 293T. The bioluminescence signal was corrected for the co-expressed copGFP fluorescence, measured separately with a plate reader. Here, copGFP levels reflect the transfection-based differences in luciferase expression due to a specific viral T2A linker in the bicistronic lentiviral plasmid providing equal expression levels of both transgenes. The normalized in vitro spectra [Fig. 1] showed differences in peak photon emission

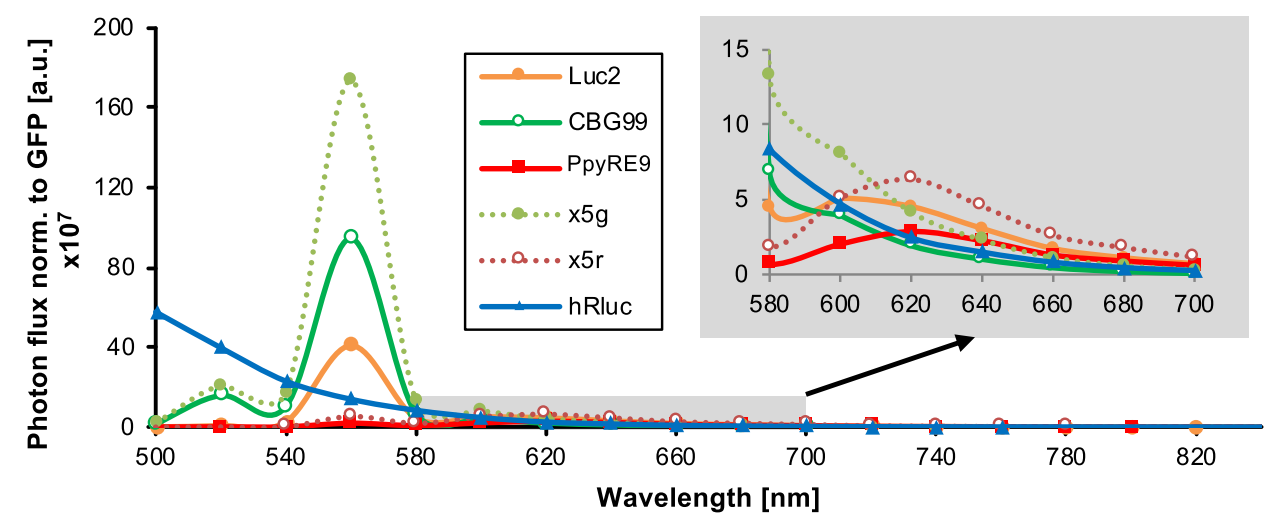

Fig. 1 Comparison of Luc2, CBG99, PpyRE9, $x 5 \mathrm{~g}, \mathrm{x} 5 \mathrm{r}$, and hRluc emission spectra in vitro. Plotted is the photon emission from EF1 $\alpha$-luciferase-T2A-copGFP transfected HEK 293T cells recorded with the IVIS Spectrum CT system (mean of $n=4$ to 6). Photon emission has been normalized to the individual copGFP expression. Graph with gray background is a zoom-in for 580 to $700 \mathrm{~nm}$ to highlight the differences in the red part of the spectra.

(a)

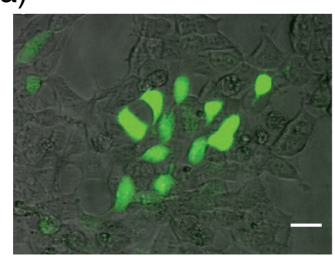

(c)

\begin{tabular}{|c|c|c|}
\hline & $x 5 g$ & $x 5 r$ \\
\hline \multirow{6}{*}{ 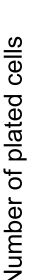 } & 0 & 600,000 \\
\hline & 200,000 & 400,000 \\
\hline & 300,000 & 300,000 \\
\hline & 400,000 & 200,000 \\
\hline & 500,000 & 100,000 \\
\hline & 540,000 & 60,000 \\
\hline
\end{tabular}

no cell control

open filter $\quad \times 5 g$ unmixing $\quad \times 5 r$ unmixing
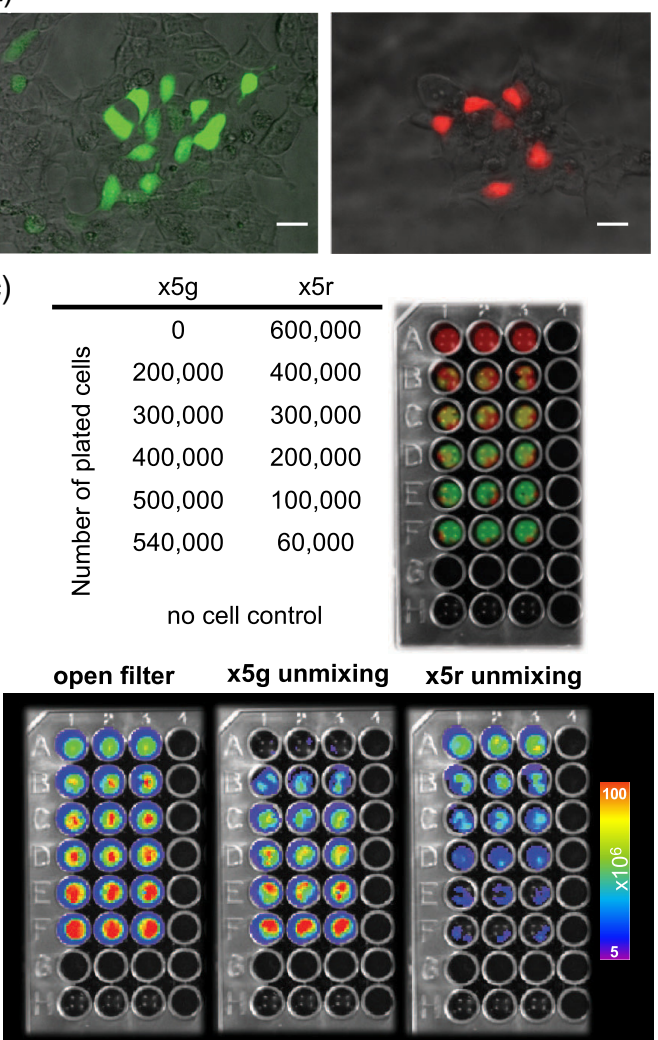

(c)

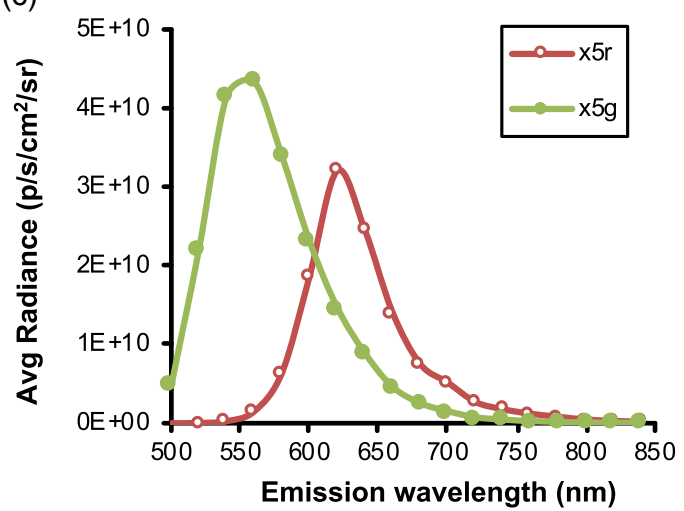

(d)

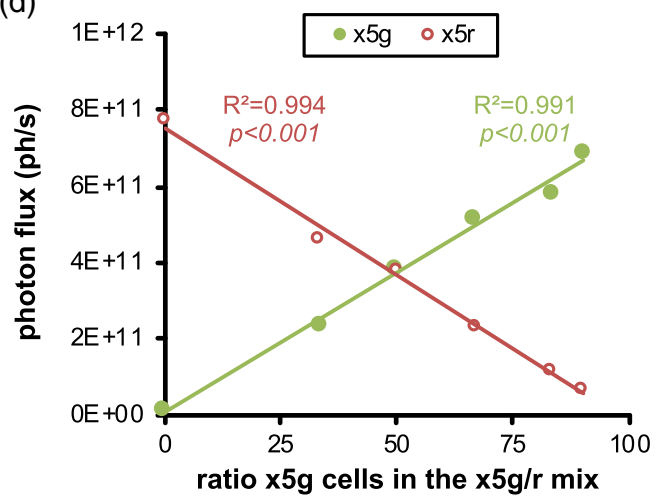

Fig. 2 Unmixing of $x 5 g$ and $x 5 r$ luciferases expressed by HEK 293T cells. (a) Fluorescence microscopy overlay with phase contrast image of cells at 2 days post transfection, used for the unmixing experiment (scale bar $25 \mu \mathrm{m}$ ). (b) Unmixed spectra of $x 5 \mathrm{r}$ and $x 5 \mathrm{~g}$ calculated by the unmixing algorithm. (c) Plating scheme for $\times 5 \mathrm{~g}$ and $\times 5 \mathrm{r}$ cells in different numbers $(n=3)$ and the corresponding false-colored unmixed results displayed as green/red composite as well as the open filter, $x 5 \mathrm{~g}$, and $\mathrm{x} 5$ red unmixed images. (d) Plot of measured unmixed $\times 5 \mathrm{~g}$ and $\times 5 \mathrm{r}$ photon flux plotted against the plated ratio of $\times 5 \mathrm{~g}$ cells in the mix $(n=3)$. Linear fitting for both luciferases underlines high accuracy of unmixed values with plated cells. 
as well as spectra shape. Notably, $\mathrm{x} 5 \mathrm{~g}$ produced in our setting the highest total photon flux (area under curve, AUC), compared to CBG99 and Luc2 (1.79- and 3.69-fold, respectively). For x5r, we found the AUC value to be 2.24-fold higher compared to PpyRE9 but only 0.45 times of the total photons from Luc2. The photon flux from cells expressing hRluc cannot be compared directly due to the different substrate (coelenterazine); however, the spectra clearly indicated the strong monophasic blue/green light emission. In contrast, emission spectra of Fluc and click beetle luciferase followed a biphasic spline and in vitro/in vivo emission maxima of $520 / 560 \mathrm{~nm}$ (x5g, CBG99), 560/620 nm (PpyRE9, x5r), and 560/600 nm (Luc2). The spectral separation was higher for $\mathrm{x} 5 \mathrm{~g} / \mathrm{x} 5 \mathrm{r}$ for all measured wavelengths, compared to the other green/red couples CBG99/ PpyRE9, x5g/Luc2, and CBG99/Luc2. The second-best separation was achieved by combining $x 5 \mathrm{~g}$ and Luc2.

As the next step, we performed spectral unmixing of HEK $293 \mathrm{~T}$ cells transfected with $\times 5 \mathrm{~g}$ or $\mathrm{x} 5 \mathrm{r}$ Fluc [Fig. 2(a)]. With the automated spectral unmixing algorithm provided by the IVIS software, the green and red emission spectra of $x 5 \mathrm{~g}$ and $\mathrm{x} 5 \mathrm{r}$ were extracted [Fig. 2(b)] and used for predicting the $x 5 \mathrm{~g} / \mathrm{x} 5 \mathrm{r}$ ratio in different mixtures [Fig. 2(c)]. The quantitative analysis of the unmixing data revealed high accuracy of predicted $x 5 \mathrm{~g}$ ratios in relation to the actual number of plated $\mathrm{x} 5 \mathrm{~g}$ cells
[Fig. 2(d)]. A linear regression analysis showed that for both spectra, the unmixed photon counts were in linear relationship to the amount of Fluc cells for both reporters, $x 5 \mathrm{~g}$ and $\mathrm{x} 5 \mathrm{r}$ $\left(R^{2}=0.991 p<0.001\right.$ and $\left.R^{2}=0.994, p<0.001\right)$. For predicting the correct $x 5 \mathrm{~g} / \mathrm{r}$ ratios, the algorithm resulted in a very low mean absolute deviation (MAD) of $0.02 \pm 0.02$.

The influence of light absorption and scattering on the $x 5 \mathrm{~g} / \mathrm{r}$ Fluc photon emission was determined with a tissue-like blood agar mix consisting of gelatin, intralipid, and hemoglobin [Fig. 3(a)]. Plated x5g and x5r cells were covered with different amounts of the blood agar mix before imaging in order to mimic cell grafts implanted at different tissue depths. With increasing blood agar volume, the number of detected photons decreased for $x 5 \mathrm{r}$ and $\mathrm{x} 5 \mathrm{~g}$ with an exponential two-phase decay [Fig. 3(b)]. The photon flux of $\mathrm{x} 5 \mathrm{~g}$ compared to $\mathrm{x} 5 \mathrm{r}$ remained on average 1.79-fold higher, independent of blood agar volume. Thus, under these in vitro conditions, no change in the $\mathrm{x} 5 \mathrm{~g} / \mathrm{r}$ emission ratio was detected and no additional correction factor for the Fluc-specific absorption was calculated.

\subsection{In Vivo Spectral Unmixing}

We determined the efficiency and accuracy of $x 5 \mathrm{~g}$ and $\mathrm{x} 5 \mathrm{r}$ Fluc unmixing in vivo by BLI of transfected HEK $293 \mathrm{~T}$ cells (a)

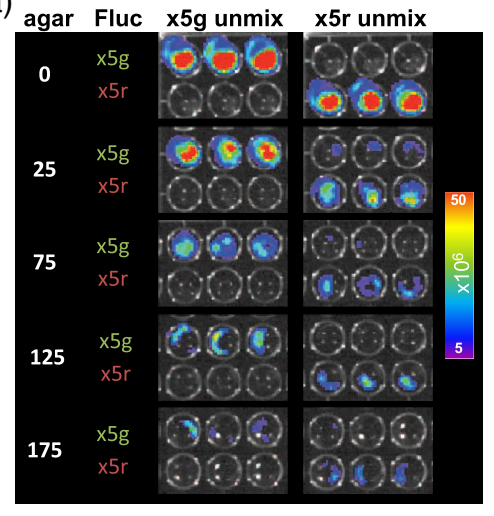

(b)

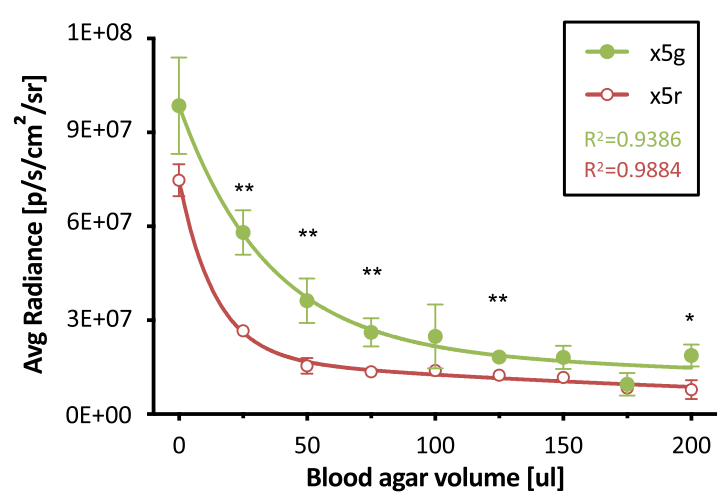

Fig. 3 In vitro modeling of superficial versus deep $x 5 \mathrm{~g}$ and $x 5$ r Fluc sources with tissue-like agarose. (a) Different volumes of tissue-like agarose are placed above the HEK 293T cell layer to mimic the overlaying tissue in different thickness. Micrographs represent selected bioluminescence/photo overlays from the $x 5 \mathrm{~g}$ and $\times 5 \mathrm{r}$ unmixing, respectively. (b) Quantitative analysis and polynomial fit for the decreasing photon emission, detected when more blood agar volume is added.

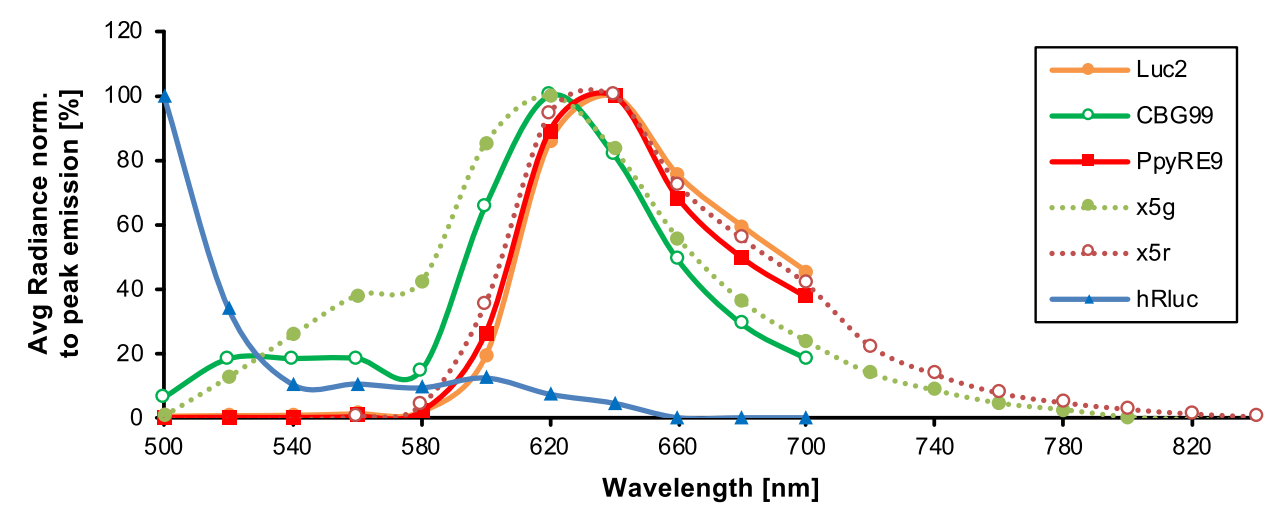

Fig. 4 In vivo emission spectra of $x 5 g$ and $x 5 r$, in comparison to Luc2, CBG99, PpyRE9, and hRluc, expressed in mammalian cells implanted in the mouse brain (data in part is adapted from our previous publication comparing different luciferases ${ }^{25}$ ). 
which were transplanted into nude mouse brains and imaged on the following day. In comparison to other luciferases, $\mathrm{x} 5 \mathrm{~g}$ and $x 5 r$ spectra exhibited substantial differences (Fig. 4). The normalized spectra of $\mathrm{x} 5 \mathrm{~g}$ revealed, in comparison to the green-shifted click beetle luciferase CBG99 at $560 \mathrm{~nm}$, a 2.07-fold higher photon flux. Thus, the green shoulder of the $x 5 \mathrm{~g}$ spectrum was much more prominent. The differences between the two red-shifted Flucs PpyRE9 and x5r in terms of total photon flux and shape of the spectrum were much less distinct.

To identify the influence of transplantation depth, we performed experiments with two separate groups: the cortical $(-1.5-\mathrm{mm}$ depth) as well as striatal $(-3.0-\mathrm{mm}$ depth) grafts. The experimental setting [Figs. 5(a) and 5(d)] included mice with $\mathrm{x} 5 \mathrm{r}$ and $\mathrm{x} 5 \mathrm{~g}$ cells only and mice that received both cell types in different mixtures. The two control mice were used (a)

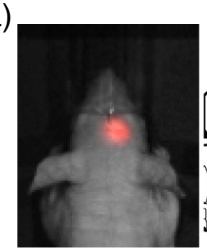

(b)

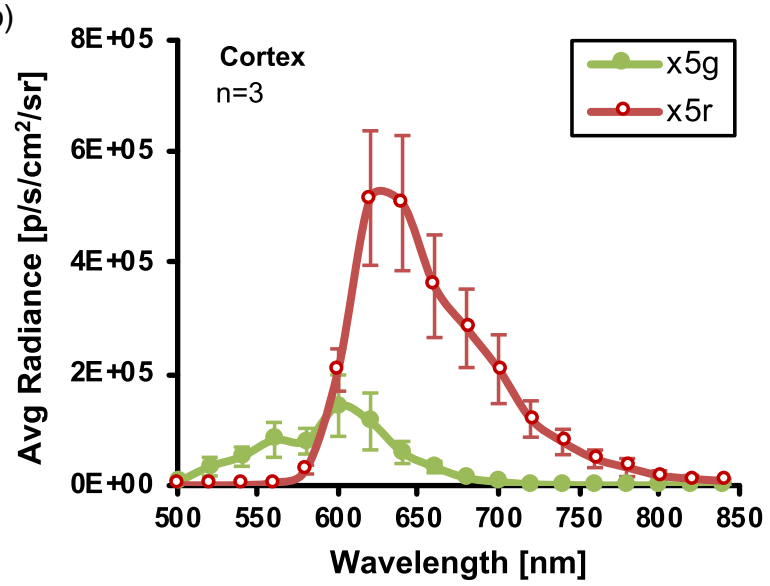

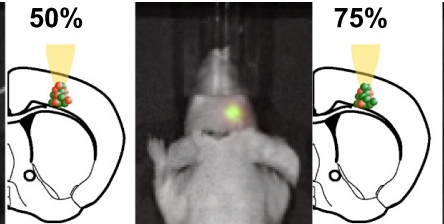

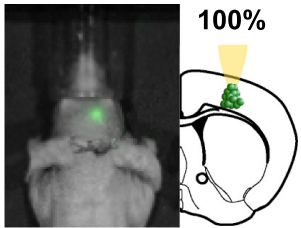

(c)

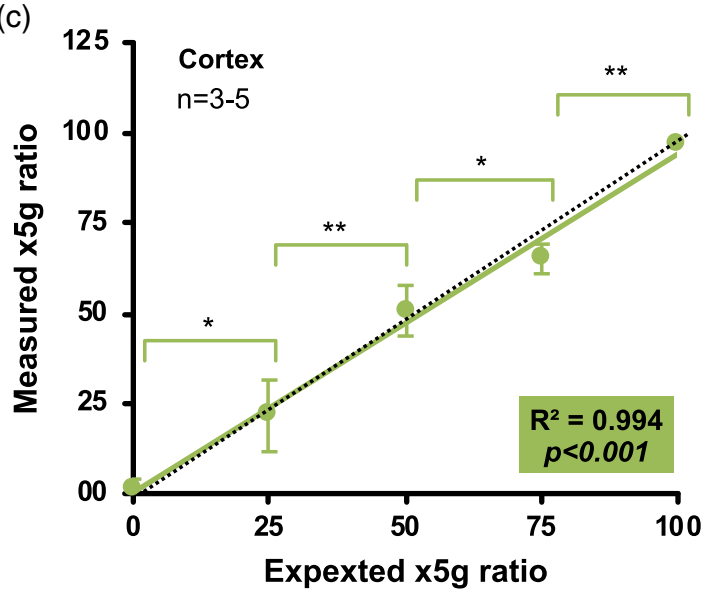

(d)
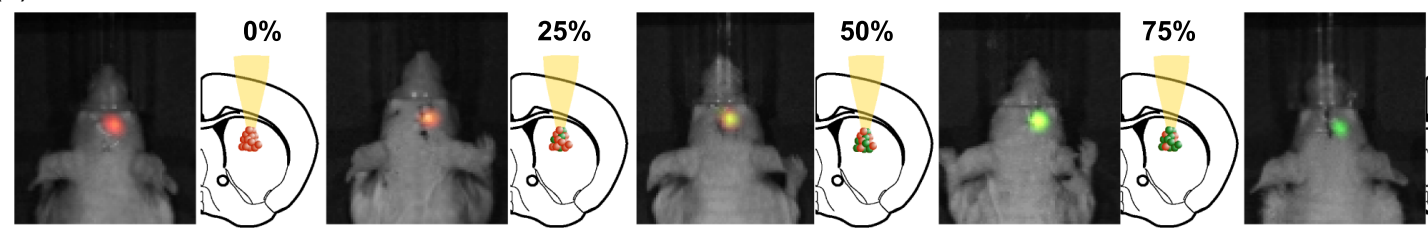

$100 \%$

(e)
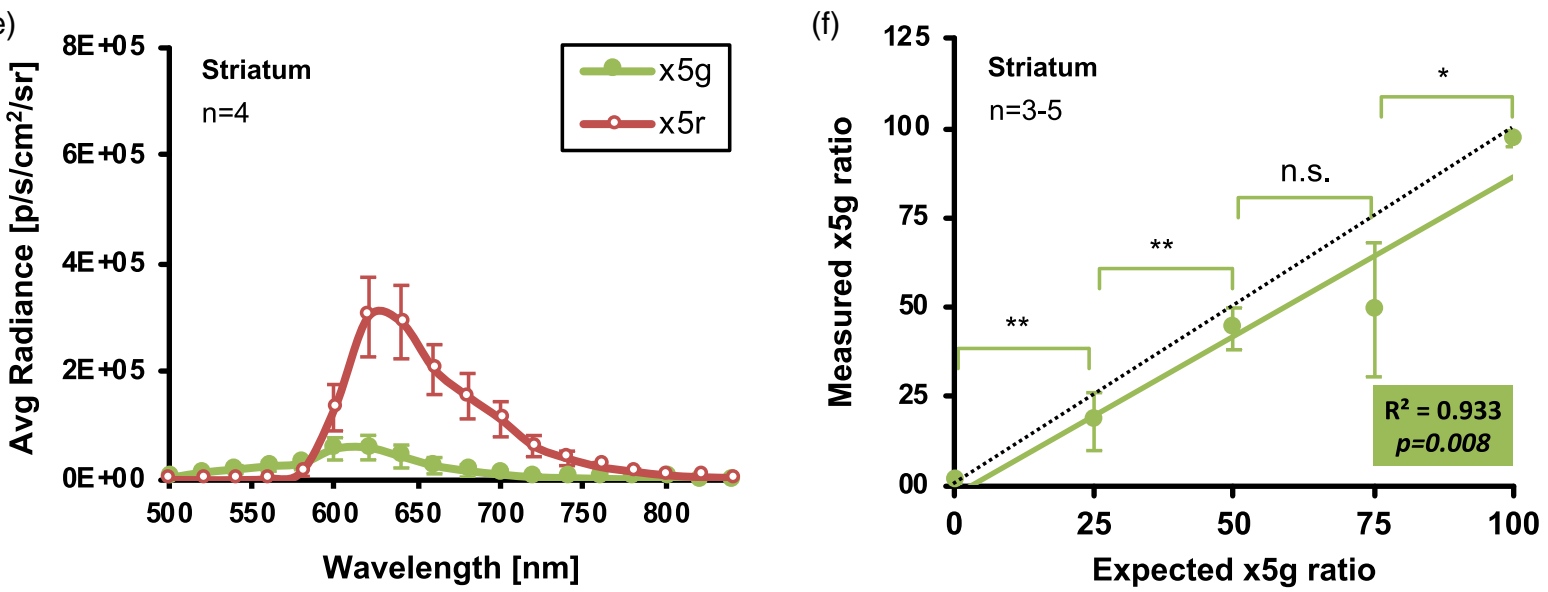

Fig. 5 Spectral unmixing efficiently determines $x 5 \mathrm{~g} / \mathrm{r}$ cell ratios in vivo. Transiently transfected HEK 293T cells expressing $x 5 \mathrm{~g}$ or $\times 5 \mathrm{r}$ luciferases are transplanted into the cortex (a) or striatum (d) and BLI is applied 1 day later. Non-normalized spectra from the $x 5 \mathrm{~g}$ and $\times 5 \mathrm{r}$ cells differ, dependent on transplantation depth: cortex (b), striatum (e). Spectral unmixing of $x 5 \mathrm{~g}$ ratio is in good agreement with expected (dotted line) $\times 5 \mathrm{~g}$ ratio for the cortical (c) as well as striatal (f) grafts. Significant differences are highlighted by asterisks. 
to extract the in vivo $\mathrm{x} 5 \mathrm{r}$ and $\mathrm{x} 5 \mathrm{~g}$ spectra from the cortex and striatum groups in the auto-unmixing mode, in which the software automatically detected x5r and x5g [Figs. 5(b) and 5(e)]. Notably, the total photon flux of $\mathrm{x} 5 \mathrm{~g}$ and $\mathrm{x} 5 \mathrm{r}$ luciferase in the cortex was 1.93-fold and 1.76-fold higher, compared to the striatum. The emission maximum of $x 5 \mathrm{r}$ in cortical and striatal grafts was $620 \mathrm{~nm}$, however reached in the striatum only $58.44 \%$ photon flux. In the case of $\mathrm{x} 5 \mathrm{~g}$, the spectrum in the striatal group was $20 \mathrm{~nm}$ shifted and the maximal photon emission was only $41.98 \%$ of the cortex group. In the cortex group, automatic unmixing of the $\mathrm{x} 5 \mathrm{~g}$ ratio worked well with high accuracy for all the expected $\mathrm{x} 5 \mathrm{~g}$ ratios $[F(4,13)=$ $217.455, p<0.001]$. A general comparison of expected to calculated $\times 5 \mathrm{~g}$ ratio resulted in a significant effect $[F(4,17)=$ 79.961, $p<0.001]$, however, with stronger linear correlation compared to the striatum $\left(R^{2}=0.994 / p<0.001\right.$ versus $R^{2}=$ $0.933 / p=0.008)$ [Figs. 5(c) and 5(f)]. A post-hoc comparison showed that, for example, the unmixing result was not precise enough to significantly separate cell grafts with $50 \%$ and $75 \%$ $\mathrm{x} 5 \mathrm{~g}$ cells, respectively $(p=0.179)$. To further calculate the accuracy of unmixing, the linear regressions for cortical and striatal grafts were statistically compared to the implanted $\mathrm{x} 5 \mathrm{~g}$ ratios [Figs. 4(c) and 4(f)]. The difference between the slopes for cortical and striatal grafts were not significantly different ( $p=0.319$ and $p=0.450)$. In addition, a one-sample $t$-test was calculated to compare the unmixing results with the known $\mathrm{x} 5 \mathrm{~g}$ ratio and MAD was calculated to forecast the error in an experiment. For cortical and striatal grafts, all unmixing ratios were not statistically significantly different except $100 \% \times 5 \mathrm{~g}$, which might be influenced by the small variation and sample size. The MAD for cortical grafts was smaller (4.5) compared to striatal grafts (8.0), supporting the observation that unmixing results and the expected $\mathrm{x} 5 \mathrm{~g}$ ratios were found to be very reliable for the cortex and less reliable for the striatum (Table 1).

We further processed the data assuming a scenario where the two control mice with $\times 5 \mathrm{~g}$ and $\mathrm{x} 5 \mathrm{r}$ cells for the auto-unmixing mode were not present. The in vivo spectra and mean attenuation factors calculated from three (cortex) or four (striatum) independent experiments were used to generate "library" spectra and to guide the unmixing algorithm [Figs. 5(b) and 5(e)]. In this case, the two control mice $\mathrm{x} 5 \mathrm{r}$ and $\mathrm{x} 5 \mathrm{~g}$ would become obsolete. However, our calculations revealed less precise spectral unmixing for cortical and striatal grafts (Table 1). In the cortex group, statistically significant differences in $\mathrm{x} 5 \mathrm{~g}$ ratios were not predicted for $25 \%$ versus $50 \%$ versus $75 \% \mathrm{x} 5 \mathrm{~g}$ cells in the mix.

Table 1 Summary of in vivo unmixing validation.

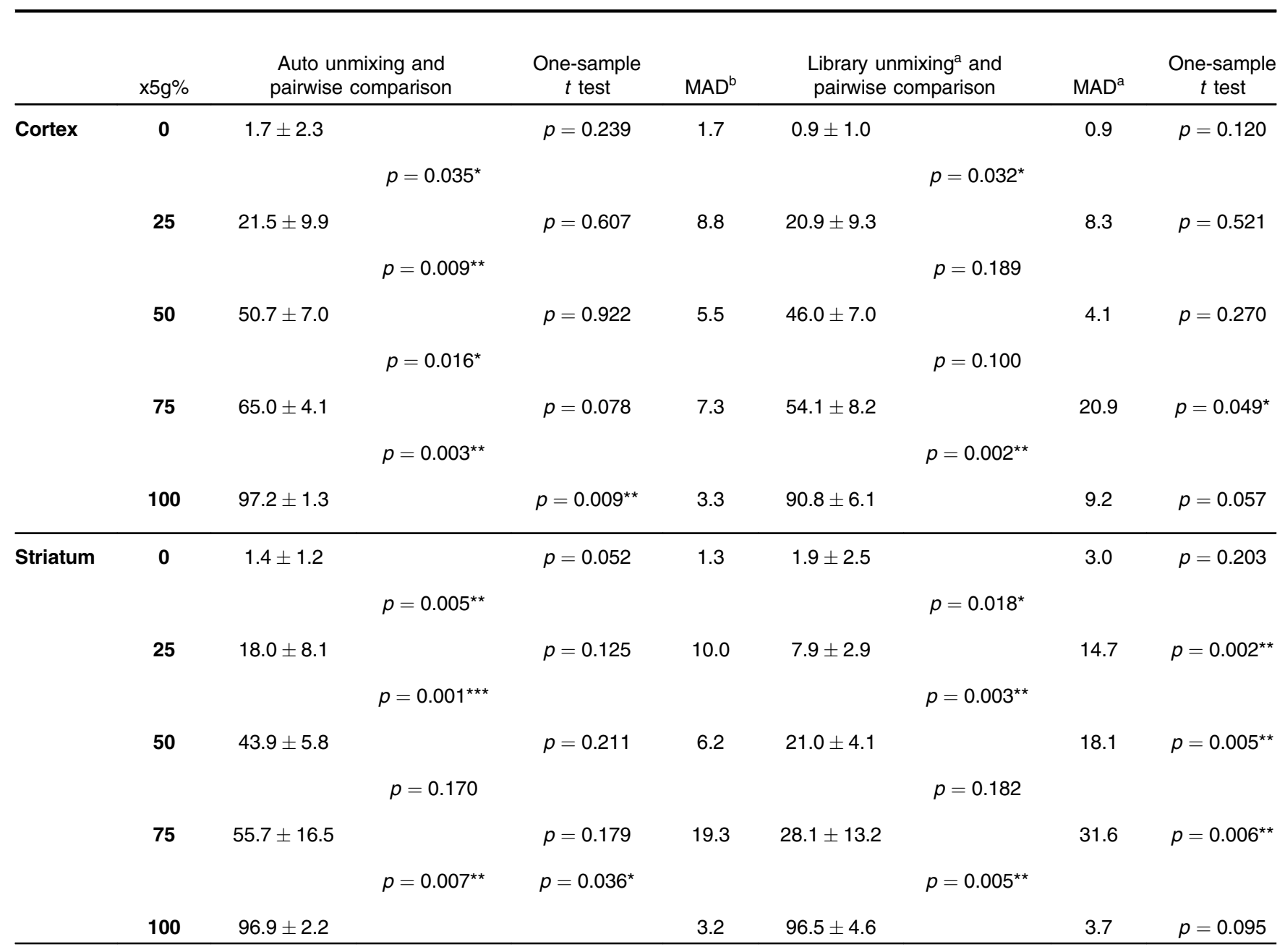

aMean absolute deviation.

${ }^{b}$ Calculated with mean attenuation factor calculated from auto unmixing experiments: cortex $\times 5 \mathrm{r} / \mathrm{x} 5 \mathrm{~g}(5.99 \pm 2.46 / 10.99 \pm 6.85)$ and striatum $\times 5 \mathrm{r} / \mathrm{x} 5 \mathrm{~g}(9.04 \pm 4.02 / 11.50 \pm 7.51)$. Statistical significance reported as $p$-value result of the $t$-test between a pair adjacent $\times 5 \mathrm{~g}$ ratios. 
Most obvious was the strong under-representation of $\mathrm{x} 5 \mathrm{~g}$ in the mix especially for the striatal grafts which led to false unmixing results, e.g., for $50 \% \mathrm{x} 5 \mathrm{~g}$ in the striatum $23.0 \% \pm 4.1 \%$, which was statistically significantly different from the implanted $50 \%$ x $5 \mathrm{~g}$ cells. Similarly, the linear relationship for the increasing ratio of $\mathrm{x} 5 \mathrm{~g}$ cells in the mix held true but with inferior correlation compared to the automated unmixing (cortex: $\mathrm{R}^{2}=0.939 / p=0.007$, striatum: $\left.R^{2}=0.856 / p=0.024\right)$.

\subsection{Discriminating Two Different Neural Stem Cell Populations}

To further probe the spectral unmixing approach, we tested $\mathrm{x} 5 \mathrm{~g}$ in combination with the widely used Fluc Luc2 in NSCs. The human H9-NSC line was stably transduced using EF1 $\alpha$-Luc2-T2A-copGFP or DCX-x5g-T2A-EGFP lentiviral vectors and sorted by FACS for their GFP expression, respectively (data not shown). The resulting two stable transgenic cell lines were transplanted into the cortex of nude mice in the same mixture ratios as for the HEK $293 \mathrm{~T}$ experiment $(0 / 100,25 / 75$, $50 / 50$, and $100 / 0 \% \times 5 \mathrm{~g} / \mathrm{r}$ ) and were imaged 1 and 2 days later [Fig. 6(a)]. The quantitative analysis revealed a linear correlation for both time points with a stronger correlation on the first day after transplantation [Fig. 6(b)]. The presence of the different cell types was verified by immunohistochemistry [Fig. 6(c)].

\section{Discussion}

We have recently characterized mutated luciferases originating from various species such as Photinus pyralis Fluc (Luc2, PpyRE9), Pyrophorus plagiopthalamus (CBG99), Gaussia princeps (Gluc), and Renilla reniformis (hRluc) for neuroimaging, which was particularly challenging, as the bioluminescent photons need to pass thick layers of bones and connective tissues. We further showed that the spectral unmixing for CBG99 and PpyRE9 was also possible for deep tissue grafts, however, with limitation to qualitative analysis. ${ }^{25}$ The aim of the present investigation was to optimize the spectral unmixing for in vivo bioluminescent neuroimaging by using advanced green- and red-shifted Fluc mutants and to characterize the accuracy to predict cell population ratios from quantitative unmixing results.

The conventional in vitro dual-luciferase assay combines Renilla luciferase and Fluc. ${ }^{26}$ These luciferases require different substrates (Fluc luciferin and Renilla coelenterazine), which makes the bioluminescent signal distinguishable. However, for many in vivo applications, the dual-luciferase approach is impractical because of the weak quantum yield, the absorptionand scattering-sensitive blue light emitted by Renilla, and it requires two separate imaging sessions. A different approach

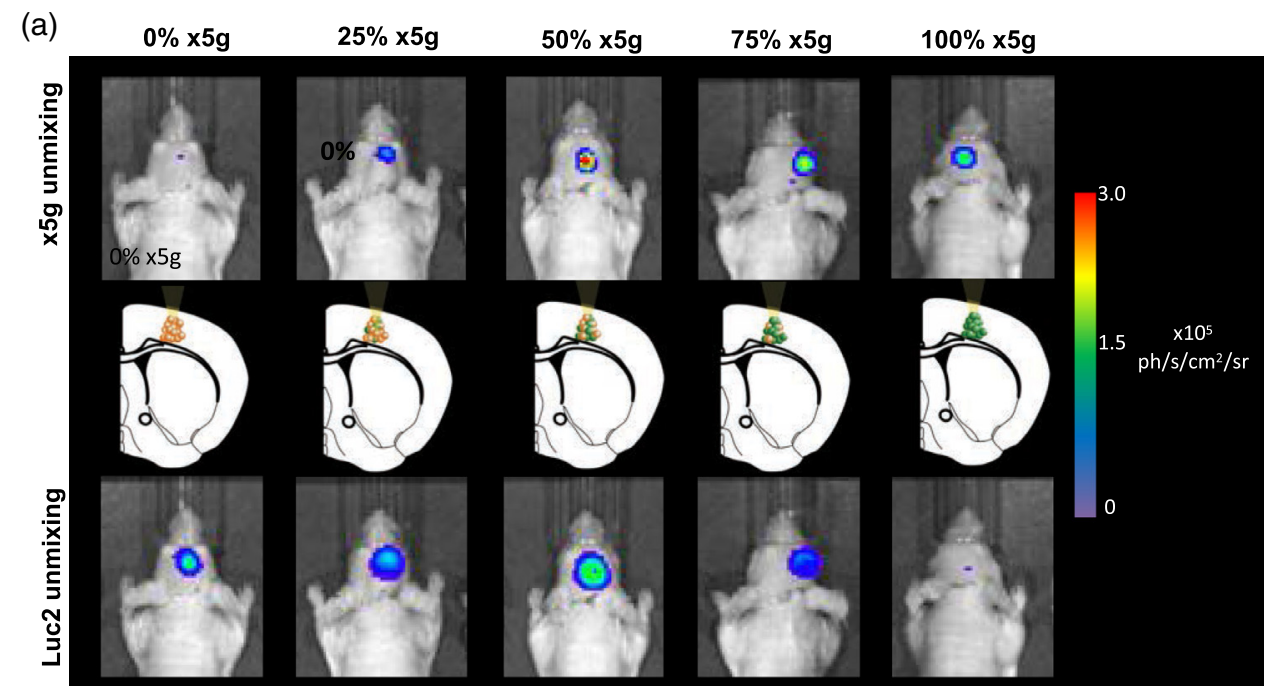

(b)

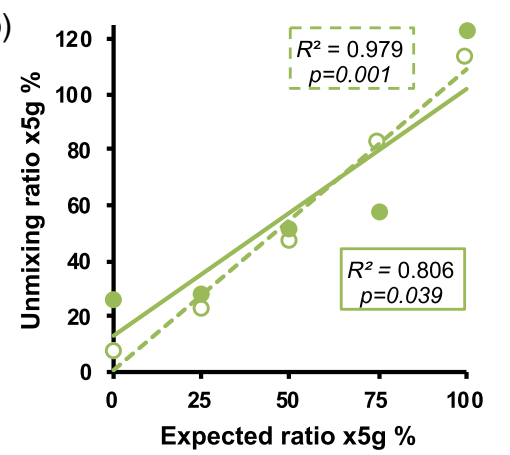

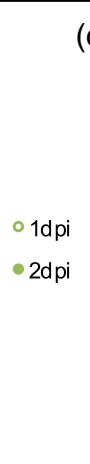

(c)

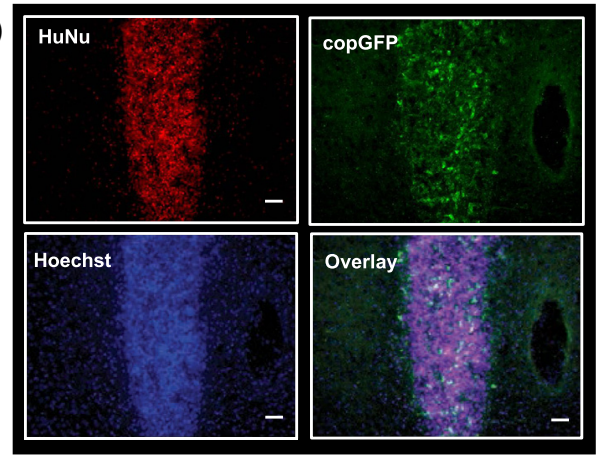

Fig. 6 Spectral unmixing of two NSC populations. (a) NSC lines expressing $x 5 \mathrm{~g}$ or Luc2 are implanted in different ratios into the cortex of nude mice. (b) Quantitative analysis of spectral unmixing from imaging data acquired 1 day and 2 days post implantation (dpi). (c) Representation of immunohistochemical staining of a NSC graft (EF1 $\alpha$-Luc2-T2A-copGFP). Human nuclei ( $\mathrm{HuNu}$ ) to discriminate the human cell graft in the mouse brain. Enhanced copGFP with anti-copGFP antibody to visualize transplanted cells expressing the transgene. Hoechst stain helps to visualize cell nuclei. Scale bar 50 um. 
is spectral unmixing, a dissecting algorithm, which is able to partition bioluminescence signals originated from multiple fluorescence or luciferase sources into individual contributors. ${ }^{16,27}$ Although spectral unmixing is widely used in fluorescence microscopy for cell imaging, ${ }^{28}$ in vivo applications-especially for neuroimaging - are technically challenging. It requires sensitive BLI detectors and engineered luciferases with distinct in vivo spectra. In a previous study, which was the first to compare reporter genes specifically for mouse brain applications, we found Fluc Luc2 to be superior to other luciferases and the quantum yield (amount of photons emitted per luciferin molecule processed) to be more important than emission wavelength. ${ }^{25}$ As we have shown here, tissue absorption and scattering have a significant impact on the luciferase spectrum, making the in vivo spectrum considerably different from that in vitro.

For our comparison, we have used the previously described red-shifted $\times 5$ mutant $^{18,19,29}$ and a novel green-shifted complement, $x 5 \mathrm{~g}$. They are based on a background that has improved thermostability, solvent stability, $\mathrm{pH}$-tolerance in terms of activity, and resistance to bathochromic shift, while retaining the same specific activity relative to the WT. ${ }^{18,22,29}$ As we could show here, $\mathrm{x} 5 \mathrm{~g}$ and $\mathrm{x} 5 \mathrm{r}$ have superior quantum yields and a better spectral separation, compared to the previously tested luciferases Luc2, PpyRE9, CBG99, and hRluc. ${ }^{25}$ The comparison of emission spectra with luciferases expressed by HEK 293T cells in vitro and in vivo highlights the strong overlap of the other green- and red-shifted luciferases with Luc2. The benefits of using $x 5 \mathrm{~g} / \mathrm{x} 5 \mathrm{r}$ for spectral unmixing compared to CBG99/ PpyRE9 are a much stronger separation due to higher photon flux and a difference in spectrum shape. Especially under in vivo conditions, the less-pronounced attenuation of the green shoulder of $x 5 \mathrm{~g}$, compared to CBG99, also favors spectral separation from deep tissue sources. Furthermore, the green-shifted $\mathrm{x} 5 \mathrm{~g}$ is 1.79- and 3.69-folds brighter than CBG99 and Luc. Likewise, $x 5 r$ is 2.24 -fold brighter than PpyRE9 ${ }^{22,23}$ and the spectrum is characterized by a higher emission for wavelengths above $600 \mathrm{~nm}$. Red-shifted probes are considered to be more efficient in vivo as light absorption in living tissue is negatively correlated with the emission wavelength. ${ }^{23,30,31}$ However, the lower quantum yield of red-shifted luciferases has so far impeded sensitive detection of cell grafts in deep tissues, such as the mouse brain. ${ }^{25}$ The tested x 5 r Fluc overcomes these limitations and is successfully validated for in vitro and in vivo spectral unmixing applications.

Under in vitro conditions, the dual-luciferase pair x $5 \mathrm{r} / \mathrm{g}$ provided the best spectral separation for all measured wavelengths, compared to previously tested red- and green-emitting luciferases. ${ }^{25}$ In this line, the unmixing of different ratios of transfected HEK cells was straightforward with a minimal MAD of $0.02 \pm 0.02$. There was a highly significant linear correlation between cell number and (unmixed) photon emission. To get reliable results, a simple correction factor compensating for the individual filter attenuation must be applied. This filter attenuation is the relation between the luciferase photon emissions without filter and the luciferase photon emissions with filter and is commonly used for in vitro dual-color luciferase assays. ${ }^{32}$ We imaged a tissue-like phantom with Fluc-expressing cells covered with a blood and fat composition of human skin to model the in vivo situation of transplanted cells in the living animal. Under these conditions, the attenuation of photon emission due to increasing amounts of blood/fat followed an exponential curve for both, $\mathrm{x} 5 \mathrm{~g}$ and $\mathrm{x} 5 \mathrm{r}$, while the difference in total photon emission remained constant ( $\mathrm{x} 5 \mathrm{~g}$ higher than $\mathrm{x} 5 \mathrm{r}$ ). According to that experiment, we did not calculate an addition correction factor. However, the in vivo emission spectra from $\mathrm{x} 5 \mathrm{~g}$ - and $\mathrm{x} 5 \mathrm{r}$ expressing HEK cells transplanted into the mouse brain revealed a strong tissue depth-dependent difference, compared to the in vitro cell culture and tissue model. Total photon counts for $\mathrm{x} 5 \mathrm{~g}$ were much lower than for $\mathrm{x} 5 \mathrm{r}$. The total $\mathrm{x} 5 \mathrm{r}$ photon counts were fivefold and 1.8-fold higher compared to $\mathrm{x} 5 \mathrm{~g}$ cells in the cortex and striatum, respectively. The $\mathrm{x} 5 \mathrm{~g}$ peak emission was affected much stronger by tissue absorption, compared to $\mathrm{x} 5 \mathrm{r}$, which was in agreement with other in vivo mouse brain studies using, for example, the red-shifted Fluc PRE9. ${ }^{30}$ Additional correction factors compensating for the Fluc-specific tissue absorption as determined for subcutaneous tumors in mice $^{33}$ would be an interesting target to further improve the quantification in future studies.

We found that the spectral unmixing algorithm works best when two "control" mice with x5g- and x5r-engrafted cells were present to allow an automatic detection of "pure" light from each luciferase. Although the emission spectra were strongly influenced by tissue absorption and scatter, statistical measures proved that for cortical grafts, with less precision for striatal grafts, quantitative unmixing was feasible. The MAD for cells implanted in the cortex of adult mice was very low (4.5) and the correlation of unmixing and actual $\mathrm{x} 5 \mathrm{~g}$ values followed a strict linear correlation which was statistically not different, providing sufficient quantitative calculations of various mixtures in dual-color imaging in vivo. In the absence of both control mice, however, the automatic unmixing algorithm tended to extract unrelated red and green components of the spectra which may lead to wrong calculations of $x 5 \mathrm{r} / \mathrm{g}$ ratio. The sampling of master spectra and an average for the filter attenuation from a batch of independent experiments resolved this problem only partially. In detail, we found less efficient unmixing for both cortical and striatal grafts under those conditions of using library data. While unmixing with the generated library data (master spectra) predicted the ratio of $x 5 \mathrm{~g}$ to be qualitatively good for cortical grafts, it underestimated $\mathrm{x} 5 \mathrm{~g}$ in the striatal grafts. We speculate that the stronger absorption of the green part of the spectrum negatively influences spectral unmixing efficiency. Nevertheless, when sampled from more experiments, the library mode could be useful for in vitro and ex vivo measurements, respectively, making controls and/or additional animals with the pure green- and red-shifted luciferases obsolete.

Confounding factors for quantitative in vivo unmixing remain the biological variability in luciferin distribution, reporter gene expression, and the stability of the image acquisition. Although the image acquisition was done in the steady state of the luciferase activity (up to $30 \mathrm{~min}$ post luciferin injection ${ }^{9}$ ), a random order of emission filters would prevent an effect of lower/higher values related to the time after substrate injection. To probe our spectral unmixing strategy in a more difficult application, we transduced human NSCs with $\mathrm{x} 5 \mathrm{~g}$ and Luc2 constructs and transplanted different ratios into the nude mouse brain. The dual-color luciferase pair $\mathrm{x} 5 \mathrm{~g} / \mathrm{Luc} 2$ was chosen as quantum yield and the spectral separation is very similar to $x 5 \mathrm{~g} / \mathrm{r}$. Furthermore, in this proof-of-concept example, the widely used luciferase Luc2 is controlled by the constitutive human $\mathrm{EF} 1 \alpha$ promoter, which maintains Luc2 levels independent of NSC maturation state. ${ }^{12}$ The $\mathrm{x} 5 \mathrm{~g}$ expression is driven by the DCX promoter and provides a second readout 
specific for early neurons. We could show that the dual-color luciferase approach is applicable to human NSCs and that spectral unmixing of in vivo BLI early after stem cell transplantation provides accurate and quantitative determination of undifferentiated cells versus pre-differentiated cells. In our experiments, the estimation of $\mathrm{x} 5 \mathrm{~g}$ ratios in the mix was as good as for the HEK 293 T cells at day 1 post implantation but slightly worse the day after. This might be due to different cell death behavior of the two cell lines or varying DCX-controlled reporter expression due to continuing neuronal differentiation. Owing to technical limitations, we have used two GFP reporters, copGFP and EGFP, for both lentiviral vectors, which cannot be distinguished by fluorescence emission. In future experiments it will be necessary to mirror the red/green luciferase also with a set of equivalent fluorescent reporter to distinguish the cell types by histology. Notably, if a direct correlation of GFP to Fluc expression were needed, a measurement of the actual protein levels and the catalytic active protein, respectively, would be necessary. ${ }^{34}$ The T2A linker regulates the bicistronic expression and not protein stability. Furthermore, spectral unmixing should be tested with other improved pairs of luciferases and luciferins such as AkaLumine- $\mathrm{HCl}^{8}$ with enhanced sensitivity for deep tissue imaging. With these technical improvements, imaging two different cell populations in the mouse brain at the same time has many important applications. In stem cell therapy of neurological disorders, for example, it could be used to monitor the viability and differentiation with two different colored luciferases in order to answer the question if the stem cell graft itself or an interplay of paracrine and immunomodulatory effects determine functional improvement.

\section{Conclusion}

Here, we have shown that spectral unmixing of dual-color bioluminescence reporter can determine the ratio of each luciferase. The unmixing is validated in human tumor and NSCs engrafted into the mouse brain cortex. Monitoring the expression of the two reporter genes by dual-color BLI holds great potential to advance luciferase-based in vitro and in vivo assays. It enables simultaneous imaging of protein and cell interaction in healthy versus disease conditions and holds the potential to advance stem cell fate imaging.

\section{Material and Methods}

\subsection{Mutagenesis}

Thermostable x5 Fluc contains five mutations (F14R, L35Q, V182K, I232K, and F465R) that reduce surface hydrophobicity and confer significant thermostability. ${ }^{20}$ The sequence of human codon-optimized WT Fluc is constructed by gene synthesis by oligo assembly and the five mutations are added by splicing by overlap extension PCR-based cloning to produce the plasmid MP5556x5, cloned into SFG-retrovirus ${ }^{35}$ upstream an internal ribosome entry site (IRES) and a truncated CD34 (dCD34) marker gene. Into this, mutations are added to produce either blue-shifted (V241I/G246A/F250S) or red-shifted (S284T) bioluminescence spectra in mutants x5green (x5g) and x5red (x5r), respectively.

\subsection{Cell Culture}

The human embryonic kidney cell line 293T (HEK 293T) was cultured in DMEM + GlutaMAX medium (Life Technologies,
Darmstadt, Germany) supplemented with 10\% FBS (Life Technologies) and 1\% penicillin/streptomycin (Life Technologies) under humidified conditions at $37^{\circ} \mathrm{C}$ and $5 \% \mathrm{CO}_{2}$. Cells were passaged every 3 days and detached with trypsin (Life Technologies). The human NSC line H9-NSC (Life Technologies), which was initially derived from the human embryonic stem cell line $\mathrm{H} 9-\mathrm{NIH}$ Registry WA09, ${ }^{36}$ was maintained according to the manufacturer's protocol. Briefly, cells were plated on Geltrex coating at a density of $5 \times 10^{4}$ cells $/ \mathrm{cm}^{2}$ in StemPro NSC SFM complete medium consisting of $1 \times$ KnockOut DMEM/F-12, $2 \mathrm{mM}$ GlutaMax, $20 \mathrm{ng} / \mathrm{mL} \mathrm{bFGF}$ and EGF, and 2\% StemPro supplement (Life Technologies). Cells were passaged every 3 days and detached with StemPro Accutase (Life Technologies).

\subsection{Tissue-like Phantoms}

Tissue-like phantom was prepared as follows: $8.76 \mathrm{mg} / \mathrm{mL}$ hemoglobin (Sigma-Aldrich) and $40 \mathrm{mg} / \mathrm{mL}$ blood agar base (Sigma-Aldrich) were dissolved in $50 \mathrm{mM}$ TBS buffer on a hotplate stirrer at $50^{\circ} \mathrm{C}$. When both components were dissolved, Lipovenös MCT (Fresenius Kabi, Bad Homburg, Germany) was added at a final concentration of $1 \%$. For the construction of the tissue phantom, the 96 -well plate with the attached transfected cells was placed on a $37^{\circ} \mathrm{C}$ warming pad, the medium was removed, and the agar mixture was layered carefully above the cells in various volumes ranging from 0 to $175 \mu \mathrm{L}$.

\subsection{Cell Transfection and Transduction}

HEK 293T cells were transfected with the plasmids pcDH-EF1 $\alpha$-x5g-T2A-tdtomato + SV40Zeo or pcDH-EF1 $\alpha$ $\mathrm{x} 5 \mathrm{r}-\mathrm{T} 2 \mathrm{~A}$-copGFP + SV40Zeo, which consisted of the constitutive promoter elongation factor 1 alpha $(\mathrm{EF} 1 \alpha)$, the genetically engineered $\mathrm{x} 5$ Fluc red $(+\mathrm{S} 284 \mathrm{~T})^{18,19,29}$ and the novel green (+V241I/G246A/F250S) mutant, the self-cleaving 2A-like peptide sequence from Thosea asigna virus (T2A), the green fluorescent protein copGFP from Pontellina plumata (Exc $482 \mathrm{~nm}, \mathrm{Em} 502 \mathrm{~nm}$ ) or the red fluorescent protein tdTomato (Exc $554 \mathrm{~nm}, \mathrm{Em} 581 \mathrm{~nm}$ ), the Simian virus 40 (SV40) origin, and a Zeocin resistance gene (Zeo). Molecular cloning of plasmids carrying Luc2, CBG99, PpyRE9, and hRluc was described previously. ${ }^{25}$ For this study, we designed novel bicistronic plasmids for constitutive and cell-specific expression of $\mathrm{x} 5 \mathrm{~g}$ and $x 5 r$ by amplifying recombinant DNA by PCR using specific primers bearing appropriate restriction sites in the following steps. At first the backbone pcDH-EF1 $\alpha$-MCS-T2A-copGFP (System Biosciences, Mountain View) was changed to pCDHEF1 $\alpha$-MCS-T2A-tdTomato (pQC NLS TdTomato IX was a gift from Connie Cepko; Addgene plasmid \#37347). In addition, we added the antibiotic resistance gene zeocin controlled by the independent SV40 promoter amplified from pBabe Zeo (pBABE zeo Ecotropic Receptor was a gift from William Hahn, Addgene plasmid \#10687). Second, $\mathrm{x} 5 \mathrm{~g}$ or $\mathrm{x} 5 \mathrm{r}$ were cloned with BamHI/NotI from M5556X5 or M5549X5 into pcDH-EF1 $\alpha$ MCS-T2A-tdtomato + SV40Zeo or pcDH-EF1 $\alpha$-MCS-T2AcopGFP+SV40Zeo. For the cell-specific plasmids, fluorescence proteins were exchanged to tdTomato and EGFP (pmEGFP-1 was a gift from Benjamin Glick, Addgene plasmid \#36409). The EF1 $\alpha$ promoter was exchanged by the human DCX promoter (kind gift of Sebastien Couillard-Despres, Paracelsus Medical University, Salzburg, Austria) via ligation with the ClaI and $\mathrm{XbaI}$ restriction sites. The final plasmids used for transduction of H9-NSCs were pcDH-EF1 $\alpha$-Luc2-T2A-copGFP and pcDH- 
DCX-x5green-T2A-EGFP. Successful cloning was verified by restriction analysis and sequencing.

For transient transfection, HEK $293 \mathrm{~T}$ cells were seeded $\left(71,400\right.$ cells $\left./ \mathrm{cm}^{2}\right)$ on gelatin-coated P60 cell culture dishes $12 \mathrm{~h}$ before transfection. Cells were transfected with $5.25 \mu \mathrm{g}$ DNA and $10.5 \mu \mathrm{L}$ Metafectene (Biontex, Munich, Germany) in $2 \mathrm{~mL}$ Opti-MEM medium (Life Technologies) and incubated for $4 \mathrm{~h}$ under normal conditions. Post transfection, cells were cultured for 2 to 3 days and prepared for in vitro dilution series or in vivo implantation.

Lentiviral vector-mediated transduction of H9-NSCs was performed using third-generation helper plasmids and subsequent cell incubation with unconcentrated pseudoviral particles for $24 \mathrm{~h}$ (for experimental details, see Ref. 12). We selected stably expressing cell lines based on the LTR-mediated background expression of EGFP and copGFP in the transduced cells via FACS (FACSAria III, BD Biosciences, San Jose).

\subsection{Cell Transplantation}

All experiments were conducted according to the guidelines laid out in the German Animal Welfare Act and were approved by the local authorities. Animals were kept in $12 \mathrm{~h} / 12 \mathrm{~h}$ day/night cycle in individually ventilated cages. Food and water were offered ad libitum.

Cells transplantation was performed as described before. ${ }^{9}$ Transfected HEK 293T cells were implanted individually or mixed (x5r:x5g; 1:1, 1:3 and 4:1), diluted with a final concentration of 150,000 cells $/ \mu \mathrm{L}$ in HBSS (Life Technologies) and stored on ice until implantation. Cells were implanted into the right striatum $(\mathrm{AP}+0.5 ; \mathrm{L}+2.0 ; \mathrm{DV}-3.0$ relative to bregma) or cortex $(\mathrm{AP}+0.5 ; \mathrm{L}+2.0 ; \mathrm{DV}-1.5$ relative to bregma) of NMRI-Foxn1nu/Foxn1nu mice (age 9 to 11 weeks, 25 to $30 \mathrm{~g}$, male from Janvier, Saint Berthevin Cedex, France). In total, we used 28 mice for cortical and 26 for striatal HEK 293 Trafts of which 6 and 3, respectively, were excluded because of visual bleeding out of the bore hole.

\subsection{In Vitro and In Vivo Imaging Setup}

BLI and fluorescence imaging experiments were performed with the IVIS Spectrum CT (PerkinElmer, Waltham). We recorded emission spectra explicitly with the IVIS system as we used the system throughout all experiments and it was shown by Zhao and colleagues previously that recording emission spectra with the IVIS System resulted in comparable spectra acquired with a spectrophotometer. ${ }^{23}$

For spectral unmixing of the in vitro dilution series, respective cell numbers were diluted in PBS (Life Technologies) and plated on a black clear-bottom 96-well plate (Sigma-Aldrich, Taufkirchen, Germany). D-luciferin potassium salt (Synchem, Felsberg, Germany) was dissolved in PBS and added in a final concentration of $1 \mathrm{mM}(\sim 0.3 \mathrm{mg} / \mathrm{mL})$. Immediately after D-luciferin application, in vitro dilution series was imaged with following settings: FOV $13.2 \mathrm{~cm}$, excitation block, emission 500 to $840 \mathrm{~nm}$ (20-nm bandpass filter) and no filter (open-filter setting), exposure time automatic, binning 8 , and f/stop 1 . In addition, copGFP fluorescence was measured using the plate reader Mithras 2 LB943 (Berthold, Bad Wildbach, Germany) with the following settings: excitation $469 \mathrm{~nm}$, emission $510 \mathrm{~nm}$, lamp energy $40 \%$, and counting time $2.0 \mathrm{~s}$. In vivo BLI was performed 1 day post implantation. We used our mouse brain-adapted imaging protocol, which is described in detail elsewhere. ${ }^{9}$
About $50 \mathrm{mg} / \mathrm{mL}$ D-luciferin sodium salt (Synchem) was dissolved in PBS, sterile-filtered, and injected intraperitoneally to each animal with a final dose of $300 \mathrm{mg} / \mathrm{kg}$ body weight before isoflurane (ISO) anesthesia (2\% ISO in $30 \% \mathrm{O}_{2} / 70 \% \mathrm{~N}_{2} \mathrm{O}$ atmosphere). Image acquisition was started $8 \mathrm{~min}$ post D-luciferin injection for $25 \mathrm{~min}$ under the following setting: FOV $22.5 \mathrm{~cm}$, excitation block, emission 500 to $840 \mathrm{~nm}$ with $20-\mathrm{nm}$ bandwidth, and open filter. The exposure time ranging from 1 to $60 \mathrm{~s}$, the lens aperture size, and the binning were set automatically by the system to reach the most sensitive setting. Finally, animals were sacrificed and brain tissues were processed for histology.

\subsection{Immunostainings}

For immunohistological staining of brain tissues, mice were transcardially perfused under ISO anesthesia with $20 \mathrm{~mL}$ PBS followed by $20 \mathrm{~mL} \mathrm{4 \%}$ phosphate-buffered paraformaldehyde (PFA). Brains were removed and frozen in $-40^{\circ} \mathrm{C}$ cold 2 -methylbutane and stored at $-80^{\circ} \mathrm{C}$. Brains were cut in $14-\mu \mathrm{m}$ thick sections in coronal plane with the cryostat (Leica, Wetzlar, Germany), mounted on object slides, and stored at $-20^{\circ} \mathrm{C}$. To equilibrate tissue, brain sections were washed three times with PBS. Antigen retrieval was performed by incubating the brain sections in $10-\mathrm{mM}$ sodium citrate buffer $(\mathrm{pH} 6.0)$ at $80^{\circ} \mathrm{C}$ for 30 min. Unspecific antibody binding was reduced by blocking with $5 \%$ normal donkey serum and $0.25 \%$ Triton X-100 in PBS for $1 \mathrm{~h}$. Primary antibodies were diluted with $0.25 \%$ Triton $\mathrm{X}-100$ in PBS and incubated overnight at $4^{\circ} \mathrm{C}$. Brain sections were stained for mouse anti-HuNu (Chemicon, 1:200) and rabbit anti-copGFP (Evrogen, 1:200). Secondary antibodiesdonkey antimouse-Cy5 (Jackson ImmunoResearch, 1:200) and donkey antirabbit-Cy3 (Jackson ImmunoResearch, 1:200) were diluted with $0.25 \%$ Triton X-100 and Hoechst 33342 (Sigma-Aldrich, 1:000) in PBS and incubated for $2 \mathrm{~h}$. Finally, brain sections were air-dried and mounted within Cytoseal XYL (Thermo Fisher Scientific).

All images were acquired with the BZ-9200 Microscope (Keyence, Neu-Isenburg, Germany). Representative images of the immunohistological staining were acquired with the $4 \times$ and $60 \times$ objectives and the respective phase contrast and fluorescence filter sets (DAPI-BP, GFP-BP, TexRed, and Cy5 HC; Keyence).

\subsection{Data Analysis}

Optical imaging data analysis and spectral unmixing was performed with the Living Image 4.3.1 software (PerkinElmer). Regions of interest with constant size were manually drawn for well plate quantification as well as for in vivo data. Total photon flux was calculated as AUC, as described previously, ${ }^{9}$ and the filter with highest average radiance was selected as emission with maximum photon flux. The normalized and non-normalized master spectra for $\mathrm{x} 5 \mathrm{~g}$ and $\mathrm{x} 5 \mathrm{r}$ in the striatum or cortex were calculated by the average of four or three different acquisitions. The master spectrum was used in the "library" mode for spectral unmixing as guideline. The automatic ("auto") spectral algorithm was used whenever possible, or replaced by the (manual) guided unmixing in which the user has to predefine where to find the pure $\mathrm{x} 5 \mathrm{~g}$ and $\mathrm{x} 5 \mathrm{r}$ spectra. The data for the unmixed populations of $x 5 \mathrm{~g}$ and $\mathrm{x} 5 \mathrm{r}$ were corrected for the experiment-specific filter attenuation factor. This factor compensated for the difference between the average radiance 
of $\mathrm{x} 5 \mathrm{~g} / \mathrm{r}$ unmixing at a certain filter (usual 600 to $620 / 620$ to $640 \mathrm{~nm}$ ) and the open-filter (without filter) setting. Furthermore, this correction factor graded the experimental variability induced by the differences during transfection and transplantation. Without correcting for filter attenuation, the unmixing results will remain qualitative. The filter attenuation factor average of all processed cortical and striatal grafts was used for the master spectrum unmixing.

Histological images were processed with ImageJ $1.48 \mathrm{~s}$ (National Institutes of Health, Bethesda). Further calculations, plotting, and statistical analysis were done with MS-Excel 2010 (Microsoft Corporation, Redmond) and SPSS 22.0 (IBM, Ehningen, Germany). For multiple group comparisons the one-way ANOVA with Welch statistics and post-hoc pairwise comparison (Tukey-corrected) was used. A $p$-value of $\leq 0.05$ was considered to be significant. A Student's $t$-test was used for the comparison of experimental groups. The onesample $t$-test was used to compare unmixing results with the implanted $\mathrm{x} 5 \mathrm{~g}$ ratio. The MAD was the difference between the actual values and the known values (implanted $\mathrm{x} 5 \mathrm{~g}$ cells). All values were expressed as mean \pm standarddeviation (SD) and statistical significance were indicated by $* p<0.05$, $* * p<0.01, * * * p<0.001$, and ${ }^{\#} \mathrm{n} . \mathrm{s}$.

\section{Disclosure}

No conflicts of interest, financial or otherwise, are declared by the authors.

\section{Acknowledgments}

We acknowledge excellent technical assistance with histology and in vivo imaging from Anja Peglow and Andreas Beyrau. This work was financially supported by grants from the EUFP7 programs TargetBraIn (HEALTH-F2-2012-279017) and BrainPath (PIAPP-GA-2013-612360). SV and MA were supported by a grant from the German research foundation DFG (AS-464/1-1). AJ acknowledges the Welsh Government for a Ser Cymru II Award (80762-CU-063).

\section{References}

1. C. E. Badr, Ed., Bioluminescent Imaging, Humana Press, Totowa, New Jersey (2014).

2. A. Rehemtulla et al., "Rapid and quantitative assessment of cancer treatment response using in vivo bioluminescence imaging," Neoplasia 2(6), 491-495 (2000).

3. D.-E. Kim et al., "Imaging of stem cell recruitment to ischemic infarcts in a murine model," Stroke 35(4), 952-957 (2004).

4. F. Sher et al., "Bioluminescence imaging of Olig2-neural stem cells reveals improved engraftment in a demyelination mouse model," Stem Cells 27(7), 1582-1591 (2009).

5. A. Tennstaedt et al., "Noninvasive multimodal imaging of stem cell transplants in the brain using bioluminescence imaging and magnetic resonance imaging," Methods Mol. Biol. 1052, 153-166 (2013).

6. C. Vandeputte et al., "Bioluminescence imaging of stroke-induced endogenous neural stem cell response," Neurobiol. Dis. 69, 144-155 (2014).

7. J. Adamczak et al., "Neurogenesis upregulation on the healthy hemisphere after stroke enhances compensation for age-dependent decrease of basal neurogenesis," Neurobiol. Dis. 99, 47-57 (2017).

8. S. Iwano et al., "Single-cell bioluminescence imaging of deep tissue in freely moving animals," Science 359(6378), 935-939 (2018).

9. M. Aswendt et al., "Boosting bioluminescence neuroimaging: an optimized protocol for brain studies," PLoS One 8(2), e55662 (2013).
10. S. Vogel et al., "Initial graft size and not the innate immune response limit survival of engrafted neural stem cells," J. Tissue Eng. Regen. Med. 12(3), 784-793 (2018).

11. J. R. de Wet et al., "Firefly luciferase gene: structure and expression in mammalian cells," Mol. Cell Biol. 7(2), 725-737 (1987).

12. A. Tennstaedt et al., "Human neural stem cell intracerebral grafts show spontaneous early neuronal differentiation after several weeks," Biomaterials 44, 143-154 (2015).

13. C. Daniel et al., "Dual-color bioluminescence imaging for simultaneous monitoring of the intestinal persistence of Lactobacillus plantarum and Lactococcus lactis in living mice," Appl. Environ. Microbiol. 81(16), 5344-5349 (2015).

14. C. A. Maguire et al., "Triple bioluminescence imaging for in vivo monitoring of cellular processes," Mol. Ther. Nucleic Acids 2, e99 (2013).

15. L. Mezzanotte et al., "A new multicolor bioluminescence imaging platform to investigate NF- $\kappa$ B activity and apoptosis in human breast cancer cells," PLoS One 9(1), e85550 (2014).

16. S. T. Gammon et al., "Spectral unmixing of multicolored bioluminescence emitted from heterogeneous biological sources," Anal. Chem. 78(5), 1520-1527 (2006).

17. S. K. Cool et al., "Comparison of in vivo optical systems for bioluminescence and fluorescence imaging," J. Fluoresc. 23(5), 909-920 (2013).

18. B. R. Branchini et al., "Red- and green-emitting firefly luciferase mutants for bioluminescent reporter applications," Anal. Biochem. 345(1), 140-148 (2005).

19. B. R. Branchini et al., "Thermostable red and green light-producing firefly luciferase mutants for bioluminescent reporter applications," Anal. Biochem. 361(2), 253-262 (2007).

20. G. H. E. Law et al., "Mutagenesis of solvent-exposed amino acids in Photinus pyralisluciferase improves thermostability and $\mathrm{pH}$-tolerance," Biochem. J. 397(2), 305-312 (2006).

21. A. Jathoul et al., "Development of a pH-tolerant thermostable Photinus pyralis luciferase for brighter in vivo imaging," in BioluminescenceRecent Advances in Oceanic Measurements and Laboratory Applications, D. Lapota, Ed., InTechOpen Limited, London (2012).

22. B. R. Branchini et al., "Red-emitting luciferases for bioluminescence reporter and imaging applications," Anal. Biochem. 396(2), 290-297 (2010).

23. H. Zhao et al., "Emission spectra of bioluminescent reporters and interaction with mammalian tissue determine the sensitivity of detection in vivo," J. Biomed. Opt. 10(4), 41210 (2005).

24. S. Bhaumik and S. S. Gambhir, "Optical imaging of Renilla luciferase reporter gene expression in living mice," Proc. Natl. Acad. Sci. U. S. A. 99(1), 377-382 (2002).

25. L. Mezzanotte et al., "Evaluating reporter genes of different luciferases for optimized in vivo bioluminescence imaging of transplanted neural stem cells in the brain," Contrast Media Mol. Imaging 8(6), 505-513 (2013).

26. M. Hampf and M. Gossen, "A protocol for combined Photinus and Renilla luciferase quantification compatible with protein assays," Anal. Biochem. 356(1), 94-99 (2006).

27. C. Ran and A. Moore, "Spectral unmixing imaging of wavelengthresponsive fluorescent probes: an application for the real-time report of amyloid beta species in Alzheimer's disease," Mol. Imaging Biol. 14(3), 293-300 (2012).

28. T. Zimmermann, "Spectral imaging and linear unmixing in light microscopy," Adv. Biochem. Eng. Biotechnol. 95, 245-265 (2005).

29. A. P. Jathoul et al., "A dual-color far-red to near-infrared firefly luciferin analogue designed for multiparametric bioluminescence imaging," Angew. Chem. Int. Ed Engl. 53(48), 13059-13063 (2014).

30. Y. Liang, P. Walczak, and J. W. M. Bulte, "Comparison of red-shifted firefly luciferase Ppy RE9 and conventional Luc2 as bioluminescence imaging reporter genes for in vivo imaging of stem cells," J. Biomed. Opt. 17(1), 016004 (2012).

31. L. Mezzanotte et al., "Sensitive dual color in vivo bioluminescence imaging using a new red codon optimized firefly luciferase and a green click beetle luciferase," PLoS One 6(4), e19277 (2011).

32. E. Michelini et al., "Spectral-resolved gene technology for multiplexed bioluminescence and high-content screening," Anal. Chem. 80(1), 260-267 (2008). 
33. S. Pesnel et al., "Quantitation in bioluminescence imaging by correction of tissue absorption for experimental oncology," Mol. Imaging Biol. 13(4), 646-652 (2011).

34. K. A. Feeney et al., "In-depth characterization of firefly luciferase as a reporter of circadian gene expression in mammalian cells," J. Biol. Rhythms 31(6), 540-550 (2016).

35. B. Philip et al., "A highly compact epitope-based marker/suicide gene for easier and safer T-cell therapy," Blood 124(8), 1277-1287 (2014).

36. S. K. Dhara and S. L. Stice, "Neural differentiation of human embryonic stem cells," J. Cell Biochem. 105(3), 633-640 (2008).

Markus Aswendt received his MSc degree in 2009 and $\mathrm{PhD}$ in neurobiology from the University of Magdeburg and the Max Planck Institute in Cologne, Germany, in 2013, under Drs. Klaus Reymann and Mathias Hoehn. Following postdoctoral training in the lab of Dr. Gary Steinberg, Stanford University School of Medicine, California, he was appointed a junior group leader in 2017 at the Department of Neurology, University of Cologne. His lab explores neuroimaging in mice to develop therapies for stroke.

Stefanie Vogel received her MSc degree and her $\mathrm{PhD}$ in biology from the University of Cologne, under Dr. Mathias Hoehn, Max Planck Institute for Metabolism Research, Cologne, Germany, in 2018. Currently, she is a postdoctoral fellow at the Technical University Dresden, Germany, in the group of Dr. Jared Sterneckert.
Cordula Schäfer is a technical assistant for molecular cloning and cell culture at the Max Planck Institute for Metabolism Research, Cologne, Germany.

Amit Jathoul received his $\mathrm{PhD}$ in biotechnology and chemical engineering from the University of Cambridge, U.K., in 2008. Following postdoctoral training with Martin Pule at the University College London, U.K., he became a research fellow in the School of Biosciences at Cardiff University, U.K., in 2016.

Martin Pule received his bachelor's degree in medicine and surgery from the University College in Dublin, Ireland, in 1995. Following a Fulbright Scholarship at the Baylor College of Medicine, Houston, he became a senior lecturer in hematology at the University of London Cancer Institute, London, U.K. He leads the optical imaging in the Center for Biological Imaging. He and his group work on engineering mammalian cells, especially T-cells, for therapeutic applications.

Mathias Hoehn received his MSc degree in 1978 and his PhD in biophysics from the University of Regensburg, Germany, in 1982. Following postdoctoral training at the University of California, San Diego, in 1984, he was appointed head of the MRI group at the technical University of Aachen in 1985. Since 1987, he has led the in-vivoNMR lab at the Max Planck Institute for Metabolism Research in Cologne, Germany, and was appointed a visiting professor in 2013 at the Leiden University Medical Center, The Netherlands. 